\title{
Seconds Per Hour
}

National Cancer Institute

\section{Source}

National Cancer Institute. Seconds Per Hour. NCI Thesaurus. Code C150899.

A rate unit expressed in seconds per period of time equal to sixty minutes. 